\title{
Comparison of Micellar and Vesicle-Based Drug Delivery Systems
}

\author{
Alyssa Amato, Clifford E. Larrabee Jr. \\ Department of Science and Health, University of Cincinnati Clermont College \\ 4200 Clermont College Dr., Batavia, Ohio, USA \\ amatoal@mail.uc.edu; cliff.larrabee@uc.edu
}

\section{Extended Abstract}

Targeted drug delivery systems deliver therapeutic agents to specific locations in the body where they can act on the diseased tissue. An effective carrier will bind the drug and remain stable while circulating in the bloodstream until it reaches the diseased site where it will release the drug. Carriers with a diameter on the order of 10 to 200 nanometers accumulate in cancerous tumors due to the enhanced permeability and retention (epr) effect. The rapid growth of tumor cells causes leakiness in the adjacent vasculature allowing small particles to pass through. Since lymphatic drainage in the tumor cells is limited, drug-loaded carriers will passively build up in the cancerous tissue. A general problem with epr is tailoring the size of the carriers to the size of the crevices in the vasculature. Undecylenic acid vesicles with diameters in the $20 \mathrm{~nm}$ to $100 \mathrm{~nm}$ range has been reported. [1] However, these vesicles have not yet been characterized as drug delivery systems. The aim of the current research is to characterize undecylenic acid vesicles as potential carriers for targeted drug delivery systems. Previous studies of micellar drug delivery model systems based on undecylenic acid have shown that while they are less than $5 \mathrm{~nm}$ in diameter [2], they are capable of binding [3] and protecting [4] hydrophobic substrate in aqueous solution at physiological $\mathrm{pH}(7.4)$ and releasing the substrate at tumor $\mathrm{pH}(<7)$ [5]. If these features can be maintained in the vesicles while increasing the diameter to more than $20 \mathrm{~nm}$, all of the components for a therapeutic drug delivery system will be in place. Further, the aqueous interior of the vesicles opens up the possible of multifunctional carriers providing simultaneous delivery of both hydrophobic and hydrophilic drugs to the targeted sites.

Here we report on the solubilization of two hydrophobic substrates by unpolymerized and polymerized sodium 10undecenoate $(\mathrm{NaU})$ vesicles and compare the results to the micellar systems [6]. Solubilization power (SP) was determined for Oil Blue $\mathrm{N}$ by visible light absorbance at $600 \mathrm{~nm}$. The results, in mmol (substrate)/mol (NaU), are $1.48 \pm$ 0.21 (unpolymerized) and $0.68 \pm 0.37$ (polymerized). Both values are approximately four times the corresponding values for the micellar systems, $0.37 \pm 0.04$ and $0.14 \pm 0.01$. The relative polarity [7] of the environment at the substrate binding site was determined for the solvatotropic probe, Nile Red, by fluorescence spectroscopy. Polymerization of the micelle produced a large change in relative polarity, from $0.58 \pm 0.02$ to $0.80 \pm 0.02$. Polymerization of the vesicle produced a much smaller effect, from $0.69 \pm 0.02$ to $0.75 \pm 0.02$.

The overall results encourage further study of the sodium 10-undecenoate vesicle system for the rational design of therapeutic drug delivery systems. The transition from micelles to vesicles increases the solubilization power fourfold which would produce more efficient delivery. Further, the lack of sensitivity of the polarity of the binding site to polymerization gives the designer flexibility in controlling degree of polymerization for stability without fear of disrupting the system/substrate environment.

\section{References}

[1] J.-H. Lee, D. Danino, and S. R. Raghavan, "Polymerizable vesicles based on a single-tailed fatty acid surfactant: a simple route to robust nanocontainers," Langmuir, vol. 25, no. 3, pp. 1566-1571, 2009.

[2] A. Amato and J. Clifford E. Larrabee, "Tuning a Model Drug Delivery System for Size and Loading Capacity," in 2nd World Congress on New Technologies, Budapest, Hungary, 2016.

[3] C. E. Larrabee Jr, M. R. Warmin, and M. J. Howard, "Clathrate Hydrate Formation and Micellization of Tetrabutylammonium 10-Undecenoate," (in English), International Journal of Theoretical and Applied Nanotechnology, vol. 2, pp. 40-45, 2014. 
[4] E. Ogle and J. Clifford E. Larrabee, "Effect of a clathrate-forming counterion on micellar solubilization," presented at the 3rd World Congress on New Technologies (NewTech'17), Rome, Italy, 2017.

[5] M. Robison, M. R. Warmin, and C. E. Larrabee Jr, "pH-Mediated Release in a Model Drug Delivery System," in Proceedings of the 2nd World Conference on Nanotechnologies (NewTech'16), Budapest, Hungary, 2016, pp. 1091-6: Avestia, 2016.

[6] C. E. Larrabee Jr, M. R. Warmin, and C. N. Iles, "Effect of Oligomerization of Sodium 10-Undecenoate on the Solubilization of a Hydrophobic Substrate," in International Conference on Nanotechnology: Fundamentals and Applications, Prague, Czech Republic, 2014, vol. 5, pp. 104-1-4: Avestia, 2014.

[7] C. Reichardt and T. Welton, Solvents and solvent effects in organic chemistry. John Wiley \& Sons, 2011. 